\title{
FACTORES DE RIESGO ASOCIADOS AL TRABAJO DE PARTO PRETÉRMINO EN GESTANTES DEL HOSPITAL UNIVERSITARIO MATERNIDAD NUESTRA SEÑORA DE LA ALTAGRACIA SEPTIEMBRE-DICIEMBRE 2018
}

\author{
Risk factors associated to preterm labor in pregnant patients in \\ teaching Hospital Maternidad Nuestra Señora de la Altagracia \\ September-December 2018
}

\section{Jorge Octavio Sánchez Orbe ${ }^{a}$, Nathali Rodríguez Vargas ${ }^{b}$, Dilenia Maxiel Batista Garabitos ${ }^{c}$, You Nine Hernández Castillo ${ }^{d}$ y Yordano Montesano ${ }^{\mathrm{e}}$}

Recibido: 6 de diciembre, 2020 • Aprobado: 4 de febrero, 2021

Cómo citar: Sánchez Orbe JO, Rodríguez Vargas N, Batista Garabitos DM, Hernández Castillo YN, Montesano Y. Factores de riesgo asociados al trabajo de parto pretérmino en gestantes del Hospital Universitario Maternidad Nuestra Señora de la Altagracia septiembre-diciembre 2018. cysa [Internet]. [citado 22 de febrero de 2022];6(1):65-70. Disponible en: https://revistas.intec.edu.do/index. $\mathrm{php} / \mathrm{cisa} /$ article/view/2404

\section{Resumen}

Objetivo: determinar los factores de riesgo asociados al trabajo de parto pre-término en gestantes del Hospital Universitario Maternidad Nuestra Señora de la Altagracia, periodo septiembre-diciembre 2018.

Método: se realizó un estudio retrospectivo, descriptivo y de corte transversal, con una muestra de 52 mujeres. Los datos obtenidos fueron procesados con el sistema Epi-info 7.0.

Resultados: el estudio muestra que entre los factores de riesgo más relevantes se encuentra la multiparidad, mostrando que el $65.4 \%$ tuvieron un número mayor a dos embarazos, de las cuales se encontró que el $52.9 \%$ tienen historia de abortos. Otros factores de riesgo asociados a partos prematuros es la enfermedad obstétrica relacionada, con mayor relevancia la anemia, con un $31.6 \%$, seguida por las infecciones urinarias con un $17.5 \%$.

\footnotetext{
a Hospital Universitario Maternidad Nuestra Señora de Altagracia, Santo Domingo, República Dominicana.

ORCID: 0000-0002-2992-4632, Correo-e: drsanchezorbe@gmail.com

${ }^{\text {b }}$ Hospital Universitario Maternidad Nuestra Seńora de Altagracia.

Correo-e: nathaliro185@gmail.com

c Hospital Universitario Maternidad Nuestra Señora de Altagracia.

Correo-e: dilebatista@gmail.com
}

\begin{abstract}
Objective: Determine the risk factors associated to preterm labor in pregnant patients in "Hospital Maternidad Nuestra Señora de la Altagracia" in Santo Domingo, SeptemberDecember 2018
\end{abstract}

Methods: It is a descriptive, retrospective, and a cross-sectional study, with a sample of 52 women. The data collected was processed with the system Epi-info 7.0

Results: The study shows that multiparity is one of the most relevant risk factors for preterm delivery, showing that $65.4 \%$ had a number equal or greater than 2 pregnancies, of which $52.9 \%$ had a history of abortion. Others risk factors related to preterm delivery is a concomitant diseases within the pregnancy, being the anemia the most relevant with $31.6 \%$ and follow up by urinary tract infections with $17.5 \%$

\footnotetext{
d Hospital Universitario Maternidad Nuestra Seńora de Altagracia, ORCID: 0000-0003-2448-2043

Correo-e: youninehernandezcastillo@gmail.com

${ }^{\text {e }}$ Hospital Universitario Maternidad Nuestra Señora de Altagracia. ORCID: 0000-0003-2338-9007

Correo-e: yordano.montesano@gmail.com
} 
Conclusión: el estudio demuestra que dentro de los factores de riesgo asociados a partos pretérminos se encuentran la anemia con un $31.6 \%$ y las infecciones urinarias con un $17.5 \%$, además el $48.1 \%$ de las pacientes tenían una edad gestacional que rondaba entre 33 y 36 semanas de gestación.

Palabras clave: parto pretérmino, prematuridad, factor de riesgo, anemia, infecciones urinarias.

\section{Introducción}

La Organización Mundial de la Salud (OMS) considera que un recién nacido antes de las 37 semanas de gestación es prematuro. La tasa global de prematuridad está alrededor del $10 \%$ de nacimientos, asignando a África y Asia Meridional los primeros lugares. Es un problema de salud pública que preocupa cada vez más, debido al aumento que muestran los indicadores de las tasas, sabiendo que influyen factores que son prevenibles y que una modificación de las conductas podría hacer una diferencia en los valores actuales. ${ }^{1,2}$

El inicio de las contracciones uterinas entre las 22 y 37 semanas, con un ritmo de 4 a 8 en un tiempo de 20 a 60 minutos, se constituye, además, como un parámetro a tomar en cuenta para definir la amenaza de parto. ${ }^{10}$

Este patrón de tiempo es importante para diferenciarlas de las contracciones de Braxton Hicks, que por lo general se presentan y cuya aparición suele ser temprana, pero no son indicativas de inicio del trabajo de parto. ${ }^{3,4-6,6,12,13}$

Molina M.7, en el año 2013, realizó un estudio retrospectivo de corte transversal, Prevalencia de parto pretérmino en pacientes con infección de vía urinarias en el área de Gineco-Obstetricia del hospital provincial general de Latacunga en el periodo junio 2011-junio 2012, con el fin de determinar la relación que existe entre la infección de vías urinarias y la prevalencia de parto pretérmino, en donde
Conclusion: The study shows that within the risk factors associated with preterm delivery, anemia is within 31.6\% and the urinary tract infections are $17.5 \%$, Also related to preterm delivery we have the gestational age which was around $33-36$ weeks in $48.1 \%$ of the preterm deliveries.

Keywords: Preterm labor, prematurity, risk factors, anemia, urinary tract infection

concluyeron que la infección de vías urinarias es una patología asociada al parto pretérmino y se da principalmente en embarazadas primigestas con edad gestacional entre 31 y 36,6 semanas, y con edades entre 20-34 años de edad. ${ }^{7}$

\section{Materiales y métodos}

Se realizó un estudio de tipo retrospectivo, descriptivo y de corte transversal, con la finalidad de determinar los factores de riesgo asociados a trabajo de parto pretérmino, en el Hospital Universitario Maternidad Nuestra Señora de la Altagracia, Distrito Nacional, República Dominicana.

El período de estudio del evento abarcó los meses de septiembre a diciembre del año 2018 y la muestra fue de 52 mujeres a través de un muestreo no probabilístico por conveniencia o intencional; el criterio de inclusión era gestantes con edad gestacional $>$ a 20 semanas $\mathrm{y}<\mathrm{de} 37$ semanas que desencadenaron un trabajo de parto espontáneo y cuyo trabajo de parto tenía > $4 \mathrm{~cm}$ de dilatación.

Los criterios de exclusión utilizados fueron:

- Partos productos de un accidente.

- Partos con edad gestacional $<20$ semanas y > o $=37$ semanas.

- Historias clínicas ilegibles, no completas o no ubicables durante el estudio.

- Partos pre-términos no asociados a trabajo de parto.
Los datos fueron recolectados mediante un formulario diseñado para la captación de la información y variables de interés, cuyas historias clínicas se encontraban archivadas en el hospital. La técnica utilizada fue revisión y registro de datos.

Posterior a su análisis, los resultados se expresaron en tablas de frecuencia absoluta y gráficos de las variables de interés.

\section{Resultados}

En relación con la edad materna se encontró en nuestro estudio que el rango de edad más relevante fue de 20-24 años asociados a parto pretérmino con un $30.8 \%$ y en rango de importancia le siguen las menores de 19 ańos con un $21.15 \%$

Tabla 1. Edad en ańos como factor de riesgo en trabajo de parto pretérmino en gestantes del Hospital Universitario Maternidad Nuestra Señora de la Altagracia septiembre-diciembre 2018

\begin{tabular}{|l|c|c|}
\hline Edad & Frecuencia & Porcentaje \\
\hline$\leq 19$ & 11 & $21.2 \%$ \\
\hline $20-24$ & 16 & $30.8 \%$ \\
\hline $25-29$ & 10 & $19.2 \%$ \\
\hline $30-34$ & 9 & $17.3 \%$ \\
\hline$\geq 35$ & 6 & $11.5 \%$ \\
\hline Total & 52 & $100 \%$ \\
\hline
\end{tabular}

Fuente: formulario para la recolección de datos de los expedientes del departamento de Ginecología y Obstetricia del Hospital Universitario Maternidad Nuestra Señora de la Altagracia.

Tomando en cuenta las diferentes edades gestacionales, se destaca la frecuencia de las etapas de prematuridad.

De los 52 casos de parto pretérmino documentados en nuestro estudio, se observó que el $48.1 \%$ corresponde al parámetro de 33-36 semanas de gestación. La prematuridad tardía o moderada es la más frecuente.
Tabla 2. Edad gestacional como factor de riesgo en trabajo de parto pretérmino en gestantes del Hospital Universitario Maternidad Nuestra Señora de la Altagracia septiembre-diciembre 2018

\begin{tabular}{|l|c|c|}
\hline Edad Gestacional & Frecuencia & Porcentaje \\
\hline $22-27$ & 4 & $7.7 \%$ \\
\hline $28-32$ & 23 & $44.2 \%$ \\
\hline $33-36$ & 25 & $48.1 \%$ \\
\hline Total & 52 & $100 \%$ \\
\hline
\end{tabular}

Fuente: formulario para la recolección de datos de los expedientes del departamento de Ginecología y Obstetricia del Hospital Universitario Maternidad Nuestra Seńora de la Altagracia.

$\mathrm{Al}$ analizar los resultados de las variables del presente estudio se observó que entre los factores de riesgo más relevantes se encuentra que un $65.4 \%$ tuvieron un número igual o mayor a dos embarazos, de las cuales se encontró que el $52.94 \%$ tienen historia de abortos.

\section{Número de gestaciones}

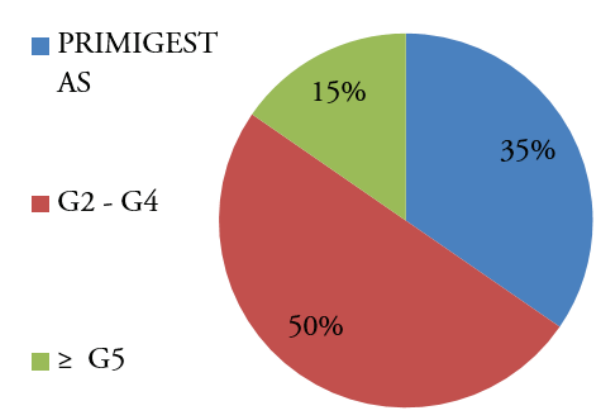

Figura 1. Número de gestaciones como factor de riesgo en trabajo de parto pretérmino en gestantes del Hospital Universitario Maternidad Nuestra Señora de la Altagracia septiembre-diciembre 2018

Fuente: formulario para la recolección de datos de los expedientes del departamento de Ginecología y Obstetricia del Hospital Universitario Maternidad Nuestra Seńora de la Altagracia. 
Otros factores de riesgo de gran importancia vinculados a partos prematuros es la enfermedad obstétrica relacionada, con mayor relevancia la anemia, con un $31.6 \%$, y seguida por las infecciones urinarias con un $17.5 \%$ de las pacientes estudiadas.

Tabla 3. Enfermedad obstétrica asociada como factor de riesgo en trabajo de parto pretérmino en gestantes del Hospital Universitario Maternidad Nuestra Seńora de la Altagracia septiembrediciembre 2018

\begin{tabular}{|l|c|c|}
\hline $\begin{array}{l}\text { Enfermedad obstétrica } \\
\text { asociada }\end{array}$ & Frecuencia & Porcentaje \\
\hline Anemia & 18 & $31.6 \%$ \\
\hline Infección urinaria & 10 & $17.5 \%$ \\
\hline RPM & 9 & $15.8 \%$ \\
\hline Infección vaginal & 7 & $12.3 \%$ \\
\hline Embarazos múltiples & 4 & $7.0 \%$ \\
\hline Pre-eclampsia & 2 & $3.5 \%$ \\
\hline HTA gestacional & 2 & $3.5 \%$ \\
\hline Apendicitis & 1 & $1.8 \%$ \\
\hline Amnionitis & 1 & $1.8 \%$ \\
\hline Oligohidramnios severo & 1 & $1.8 \%$ \\
\hline Ninguna & 0 & $0 \%$ \\
\hline Total & 52 & $100 \%$ \\
\hline
\end{tabular}

Fuente: formulario para la recolección de datos de los expedientes del departamento de Ginecología y Obstetricia del Hospital Universitario Maternidad Nuestra Señora de la Altagracia.

\section{Discusión}

En el período de septiembre a diciembre del año 2018 fueron realizados un total de 4,816 partos en el Hospital Universitario Maternidad Nuestra Señora de la Altagracia, de los cuales 461 fueron partos pretérmino, lo que representa el $9.6 \%$ de los casos. Nuestra población corresponde a 129 pacientes que desencadenaron un trabajo de parto pretérmino y espontáneo, de estos se tomó una muestra de 52 mujeres.
Comparando este total con el informe que realizó el Ministerio de Salud Pública en el año 2014, reporta que en el Hospital Universitario Maternidad Nuestra Señora de la Altagracia se registra un total de $36.0 \%$ de nacimientos prematuros. ${ }^{8}$ Teniendo en cuenta que el estudio realizado por el Ministerio de Salud Pública fue de un año completo y aplican múltiples factores de nacimientos prematuros.

Al analizar los resultados de las variables del presente estudio se observó que entre los factores de riesgo más relevantes se encuentra que un $65.4 \%$ tuvieron un número mayor a dos embarazos, de las cuales se encontró que el $52.94 \%$ tienen historia de abortos, lo cual se correlaciona con el estudio de Tucker C, Berrien K, et al. (Estados Unidos, 2015). Los factores de riesgo más fuertes con los Odds Ratios que van desde 2,34 hasta 10,78 fue el embarazo múltiple.

Otros factores de riesgo de gran importancia relacionados a partos prematuros es la enfermedad obstétrica relacionada, con mayor relevancia la anemia, con un $31.6 \%$, y le siguen las infecciones urinarias, con un $17.5 \%$ de las pacientes estudiadas. Los resultados difieren del estudio de Coiscou, Clarck y Charitable, en el año 2013, obteniendo con mayor valor las infecciones vaginales con un $44.5 \% .^{10}$

Los controles prenatales tienen una gran incidencia en el pronóstico del embarazo, se observó que un $36 \%$ no especifica cuántos números de chequeos prenatales poseían, aumentando de esta manera la posibilidad de un parto pretérmino. Las que sí se realizaban un chequeo prenatal lo hacían de forma extrahospitalaria, con un $49 \%$ de las pacientes de nuestro estudio. Disminuyendo el control gineco-obstetra con el paciente al momento del parto. En relación con el estudio realizado en el año 2015-2016 por el Dr. Javier Alexander Rivera Salas. ${ }^{3}$ Se determinó que la causa más frecuente de parto pretérmino, con un $64.1 \%$, fue la falta de controles prenatales.
En relación con la edad materna, se encontró en nuestro estudio que el rango de edad más relevante fue de 20-24 años asociados a parto pretérmino, con un $30.8 \%$; en rango de importancia le siguen las menores de 19 ańos con un $21.15 \%$.

En relación con el estudio de Coiscou, et al. ${ }^{10}$, las autoras tuvieron una gran similitud, con un resultado de $33.5 \%$ de las madres que tenían edades comprendidas entre 20-24 años.

Tomando en cuenta las diferentes edades gestacionales, se destaca la frecuencia de las etapas de prematuridad. De los 129 casos de parto pretérmino documentados en nuestro estudio, se observó que el $48.1 \%$ corresponde al parámetro de 33-36 semanas de gestación. La prematuridad tardía o moderada es la más frecuente. Resultados obtenidos por la Organización Mundial de la Salud, en un informe de la Revista Chilena de obstetricia y ginecología, Mendoza Tascón, et al., en el año 2016. Reportan unos $84,3 \%$ de casos moderadamente prematuros, conjunto a un subgrupo denominado, prematuros tardíos (34-36 semanas). ${ }^{11,15}$

El estado civil de las pacientes en nuestro estudio evidenció que un $59.6 \%$ se encontraban en unión libre, mientras que el $36.5 \%$ eran solteras. Mientras que Coiscou et al., obtuvieron que un $34.8 \%$ mantenían un estado civil en unión libre. ${ }^{10}$

\section{Conclusiones}

Una vez analizado los datos recopilados, para proseguir con el desarrollo de esta investigación, podemos dar a conocer las siguientes conclusiones:

Dentro de los factores de riesgo asociados a los partos pretérmino se encontraron la anemia, con un $31.6 \%$, las infecciones urinarias, con un $17.5 \%$, la ruptura prematura de membrana, con un $15.8 \%$, las infecciones vaginales, con un $12.3 \%$, los embarazos múltiples, con un $7 \%$, la pre-eclampsia, diabetes gestacional y la hipertensión gestacional, todas con un $3.5 \%$, y, por último, la apendicitis, la amnionitis y el oligohidramnios severo, todas con un $1.8 \%$.

El $30.7 \%$ de las pacientes tenían edades comprendidas entre los 20 y 24 años de edad.

El $48.1 \%$ de las pacientes tenían una edad gestacional que rondaba entre 33 y 36 semanas de gestación.

De las pacientes estudiadas con dos o más embarazos, el $52.9 \%$ mostró como antecedente obstétrico al menos un aborto previo, comparadas con $47.1 \%$ de las cuales no contaba con historia de abortos.

El $36.5 \%$ de las pacientes no especificaron la cantidad de controles pre-natales que tuvieron durante su embarazo al momento de ser admitidas, pero un $32.7 \%$ de las pacientes informó que habían tenido de 4 a 7 controles prenatales.

\section{Referencias bibliográficas}

1. Acosta, D. Prematuridad recién nacido es investigada por entidades. El Día [en línea]. 2018. [05 de febrero de 2018]; Disponible en: http://eldia. com.do/prematuridad-recien-nacidos-es-investigada-por-entidades/

2. Organización Mundial de la Salud. Nacimientos prematuros. [En linea].; 2018 Available from: https://www.who.int/es/news-room/fact-sheets/ detail/preterm-birth [citado el 25 de julio de 2018]

3. Rivera Salas, JA. (2016) Factores maternos y fetales asociados al parto pretérmino en pacientes atendidas en el Hospital San Vicente de Paúl de la ciudad de pasaje. [Tesis Pregrado]. Loja- Ecuador: Universidad Nacional De Loja. Área De La Salud Humana. Carrera De Medicina Humana.

4. Espinos J. Fisiopatología del Síndrome de Parto Pretérmino. Rev Per GinecolObstet, 2008;54: $15-21$ 
5. Palencia A. Parto Prematuro. Precop SCP, 2010; 9(4):10-9.

6. Pohlmann FC, Kerber N, da Silva VJ, Carvalho V, Costa C, Souza C. Premature birth: approches presents in national and international scientific production. Enfermería Global, 2016 abril;(42).

7. Molina M. (2013). Prevalencia de parto pretérmino en pacientes con infección de vias urinarias en el área de Gineco-Obstetricia del hospital provincial general de Latacunga en el periodo junio 2011- junio 2012. [Tesis de Bachiller de Medicina] Ambato, Ecuador. Universidad Técnica de Ambato.

8. Bishop EH. Pelvic scoring for elective induction. ObstetGynecol, 1964;24:266. 4 Crane JM. Factors predicting labor induction success: a critical analysis. ClinObstetGynecol 2006;49: 573-84.

9. Brown H. Manual MSD. [En línea].; 2015 Disponible en: https://www.msdmanuals.com/ es-do/professional/ginecolog\%C3\%ADa-y-obstetricia/trabajo-de-parto-y-parto-normales/ manejo-del-trabajo-de-parto-normal. [citado el 25 de julio de 2018].

10. Coiscou, A. Clark, N. Charitable, F. Factores de Riesgo Asociados a Parto Prematuro, Hospital de la Mujer Dominicana del IDSS; 2013.
11. Acuña C. Informan que en 10 meses 4,319 adolescentes han dado a luz en Maternidad La Altagracia. Salud y Soc Civil. [Seriada en línea] 2013 [Citado 2018 Agos 7]. Disponible en: http://saludysociedadcivil.org.do/index.php/ actualidad/noticias/item/477-informan-que-en10-meses-4-319-adolescentes-han-dado-a-luzen-maternidad-la-altagracia

12. Schwarcz R, Fescina R, Duverges C. Obstetricia. 6th ed.: Editorial El Ateneo; 2005.

13. Manual CTO Ginecología y Obstetricia. (6ta ed.). Ecuador.

14. Zaragoza G, Molina G, Carrillo B, et al. Marcadores Bioquímicos y Determinantes Genómicos y Proteomicos del Parto Pretérmino. [Online]. Disponible en: http://www.hvn. es/servicios_asistenciales/ginecologia_y_obstetricia/ficheros/08marcadoresbioquimicosydeterminantesezaragoza.pdf [Consultado 06 de julio 2018].

15. Poll JA, Rueda NM, Ramos H L, Poll RA, Campos BI. Riesgo cardiovascular e hipertensión arterial crónica en embarazadas. MEDISAN, 2013;17(2). 